\title{
Search for TeV decaying dark matter from the Virgo cluster of galaxies
}

\section{The HAWC Collaboration}

(a complete list of authors can be found at the end of the proceedings)

E-mail: nisamehr@msu.edu, jpharding@lanl.gov

Galaxy clusters' dynamics constitute a major piece of evidence for the existence of dark matter in astrophysical structures. The decay or annihilation of dark matter particles is hypothesized to produce a steady flux of very-high-energy gamma rays correlated with the direction of a cluster of galaxies. The Virgo cluster, being only $16 \mathrm{Mpc}$ away and spanning several degrees across the sky is an excellent target to search for signatures of particle dark matter interactions. The High Altitude Water Cherenkov (HAWC) observatory, due to its wide field of view and sensitivity to gamma rays at an energy-scale of $300 \mathrm{GeV}-100 \mathrm{TeV}$ is well-suited to perform the aforementioned search. We perform a search from the Virgo cluster for gamma-ray emission, assuming various dark matter sub-structure models using 1523 days of HAWC data. Our results provide the strongest constraints on the decay life-time of dark matter for masses above $20 \mathrm{TeV}$.

Corresponding authors: Mehr Un Nisa ${ }^{1 *}$, Pat Harding ${ }^{2}$

${ }^{1}$ Michigan State University

${ }^{2}$ Los Alamos National Labs

${ }^{*}$ Presenter

$37^{\text {th }}$ International Cosmic Ray Conference (ICRC 2021)

July 12th - 23rd, 2021

Online - Berlin, Germany 


\section{Introduction}

Over $80 \%$ of all the mass in the universe is known to consist of invisible non-baryonic matter known as dark matter (DM) [1-3]. However, despite decades of experimental searches, the particle composition of DM remains a puzzle. Astrophysical observations can complement direct detection and collider searches for DM by looking for signatures of DM annihilation or decay from regions of high DM density in the universe. Galaxy clusters with their large masses $\left(10^{12}--10^{15} \mathrm{M}_{\odot}\right)$ are amongst the most suitable candidates to search for signals of decaying DM. In this work, we consider the nearby Virgo cluster of galaxies as a possible site of DM decay. We search for gamma rays produced as a result of DM decaying via different quark and bosonic channels from an extended region of the sky spanning $8^{\circ}$ centered at the Virgo cluster, and place constraints on the decay lifetime of TeV DM.

\section{Detector and Data-set}

The HAWC observatory is a $22,000 \mathrm{~m}^{2}$ array of water tanks instrumented with four photomultiplier tubes (PMTs) each. It is located at an altitude of $4100 \mathrm{~m}$ above sea-level and continuously monitors two-thirds of the sky every 24 hours. The detector is sensitive to air-showers produced by gamma rays with energies above $300 \mathrm{GeV}$ and up to $100 \mathrm{TeV}$. The data used in this work uses the same methodologies and cuts described in Refs. $[4,5]$.

\section{Analysis}

The Virgo Cluster spans about $8^{\circ}$ on the sky and consists of multiple galaxies. The Virgo Cluster itself contains three sub-clusters. Two of these sub-clusters, Virgo-A and Virgo-B, are centered at M87 and M49. The other sub-cluster is centered around M86, an elliptical galaxy which is a galaxy merging with M87 [6]. M87, with a total mass of $2.1 \times 10^{14} M_{\odot}$, assuming a radius of $1.5 \mathrm{Mpc}$ for the center of the subcluster A, is a super-massive elliptical galaxy that is close to the center of the Virgo Cluster. The Active Galactic Nucleus (AGN) at the center of M87 is a source of $\mathrm{TeV}$ gamma rays and has been observed to go through active periods called flares [7]. M49 is the brightest elliptical galaxy in the Virgo Cluster and its total mass is about $8.7 \times 10^{13} M_{\odot}$, assuming a radius of $0.75 \mathrm{Mpc}$ for the center of the sub-cluster [6]. These two objects constitute a large fraction of the the Virgo Cluster by mass. The large apparent size and the structure of the cluster make it difficult to search for DM decay and annihilation signals via Imaging Air Cherenkov telescopes (IACTs). In addition, possible astrophysical gamma rays from the galaxies within the cluster may contaminate the analysis.

The HAWC observatory's wide FOV is well-suited to search for gamma-ray emission from extended regions in the sky. For the Virgo Cluster region, we don't detect any statistically significant gamma-ray excess due to DM with the HAWC data (Fig. 1). Therefore, we calculate lower limits on the decay lifetime of DM. 


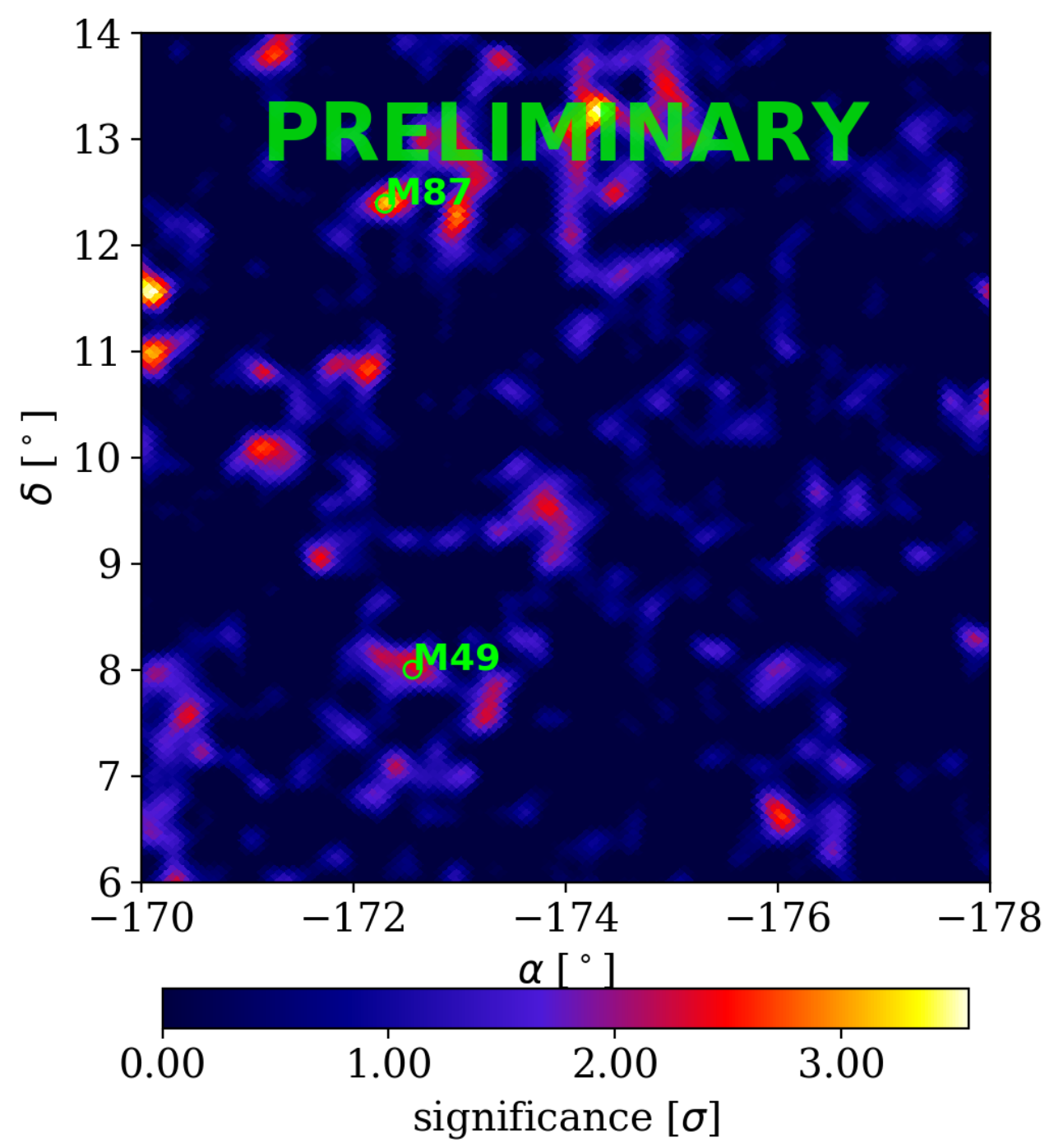

Figure 1: The point-source significance map around the Virgo cluster region using 1523 days of HAWC data with pass 4 reconstruction and the methods used in Ref. [5]. The positions of the two main sub-clusters used in this analysis M87 and M49 are labelled.

\subsection{Spectral and Spatial Models}

The expected gamma ray flux from decaying DM is given by,

$$
\frac{d \phi}{d E}=\frac{1}{4 \pi \tau M_{\chi}} \frac{d N}{d E} D
$$

where $\tau$ is the decay lifetime of DM, $M_{\chi}$ is the DM mass, $d N / d E$ is the gamma-ray spectrum 
per DM decay, and D is known as the D-factor encoding the spatial distribution of DM in the target of interest. It is defined as the integral of the DM density $\rho_{\mathrm{DM}}$ along the line-of-sight (1.o.s.) and over the solid angle $\Delta \Omega$,

$$
D=\int_{\Delta \Omega} \int_{\text {1.o.s. }} \mathrm{d} \Omega \mathrm{d} s \rho_{\mathrm{DM}}[r(s, \Omega)] \text {. }
$$

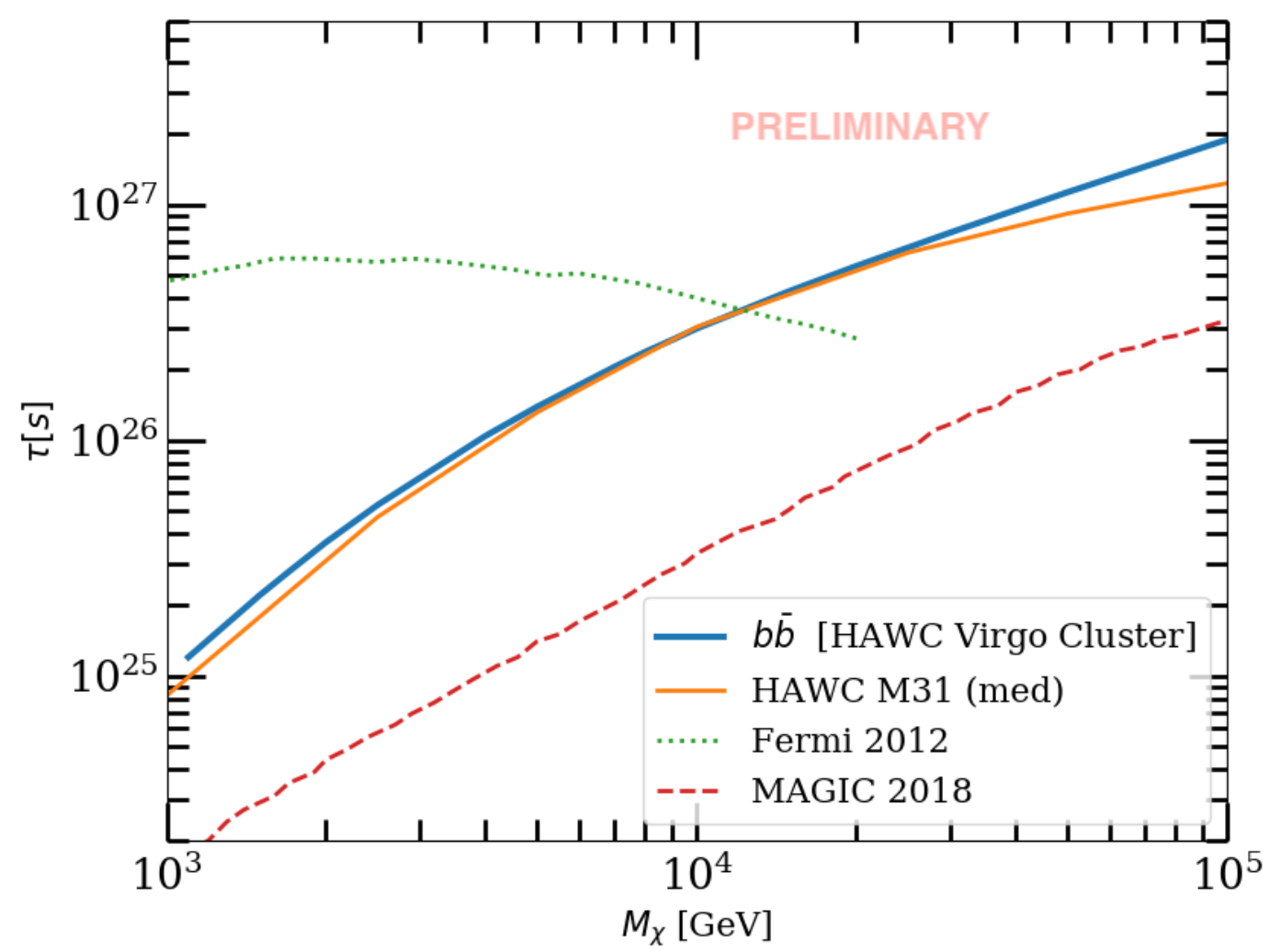

Figure 2: HAWC 95\% CL limits on the decay lifetime of dark matter decaying to $b \bar{b}$, assuming the spatial template parameters described in the text. Also shown are limits obtained using the M31 galaxy as a target with HAWC [8], Fermi-LAT [9] and MAGIC [10].

We consider DM decay to three different channels: $b \bar{b}, \tau \bar{\tau}$ and $W^{+} W^{-}$. The expected photon spectrum each channel is obtained using the publicly available "Particle Physicist Cookbook" PPPC [12] for DM masses between $1 \mathrm{TeV}$ and $100 \mathrm{TeV}$.

The DM halo for galaxies consist of a main halo, that has a smooth distribution within the galaxy, and substructure, that is attributed to the gravitationally clumped over-densities in the main halo. We construct a spatial template for the Virgo cluster as a combination of the DM templates for M87 and M49 using the software package CLUMPY [13].

Each generated template encompasses a region of interest with radius $7^{\circ}$ and the combined M87M49 template covers $10^{\circ}$ in right ascension and $12^{\circ}$ in declination. To generate these templates, we define the parameters for the underlying DM distribution by referring to the main halo and substructure properties inferred by the velocity profiles of the stars in the galaxies and N-body 


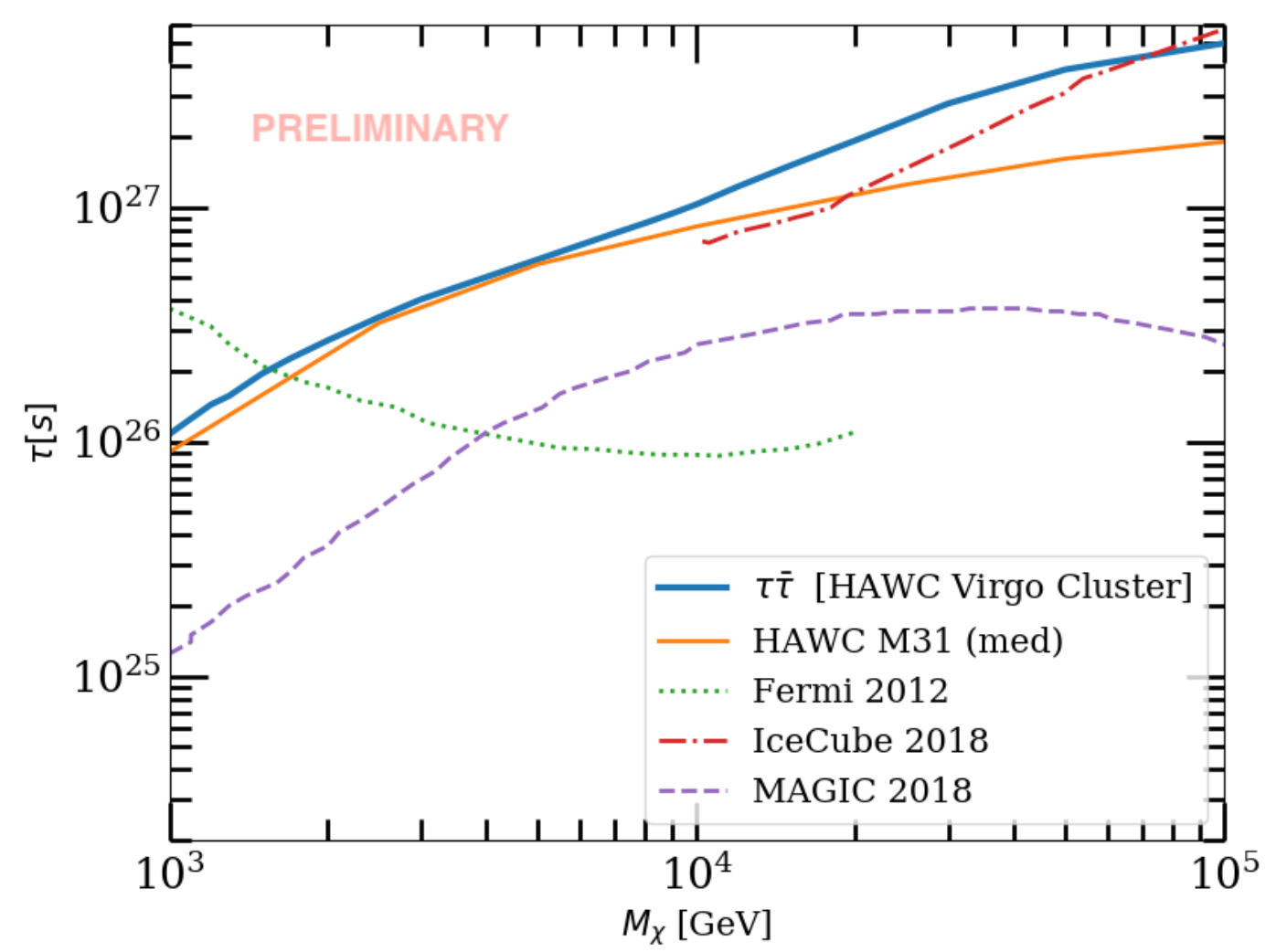

Figure 3: HAWC 95\% CL limits on the decay lifetime of dark matter decaying to $\tau \bar{\tau}$, assuming the spatial template parameters described in the text. Also shown are limits obtained using the M31 galaxy as a target with HAWC [8], Fermi-LAT [9], MAGIC [10] and IceCube [11]

\begin{tabular}{|l|l|l|l|l|l|}
\hline Object & $\begin{array}{l}\text { Distance } \\
{[\mathrm{Mpc}]}\end{array}$ & $z$ & $\mathrm{R}_{\text {vir }}[\mathrm{kpc}]$ & $\begin{array}{l}\rho_{s} \\
{\left[\mathrm{M}_{\odot} / \mathrm{kpc}^{3}\right]}\end{array}$ & $\mathrm{r}_{s}[\mathrm{kpc}]$ \\
\hline M87 & 17.2 & 0.00428 & 1700 & $6.96 \times 10^{5}$ & 403.8 \\
M49 & 17.1 & 0.00327 & 880 & $1.41 \times 10^{6}$ & 157.7 \\
\hline
\end{tabular}

Table 1: The various properties of M87 and M49 used in the construction of D factors. Columns 2-6 list the distance, redshift, virial radius, scale density and scale radius respectively.

simulations [14]. For the distribution of DM in the main halo and sub-haloes we use the generalized Navarro-Frenk-White (NFW) profile [15], with the values of free parameters fixed following Refs. [16]. The mass concentration within the sub-haloes, we adopt the model in Ref.[17, 18]. Other characteristic properties of M87 and M49 used in the simulations are listed in table 1.

\subsection{Likelihood Fitting}

We perform a likelihood maximization to place lower limits on the decay lifetime of DM corresponding to every decay channel and DM masses sampled between $1 \mathrm{TeV}$ and $100 \mathrm{TeV}$. The 


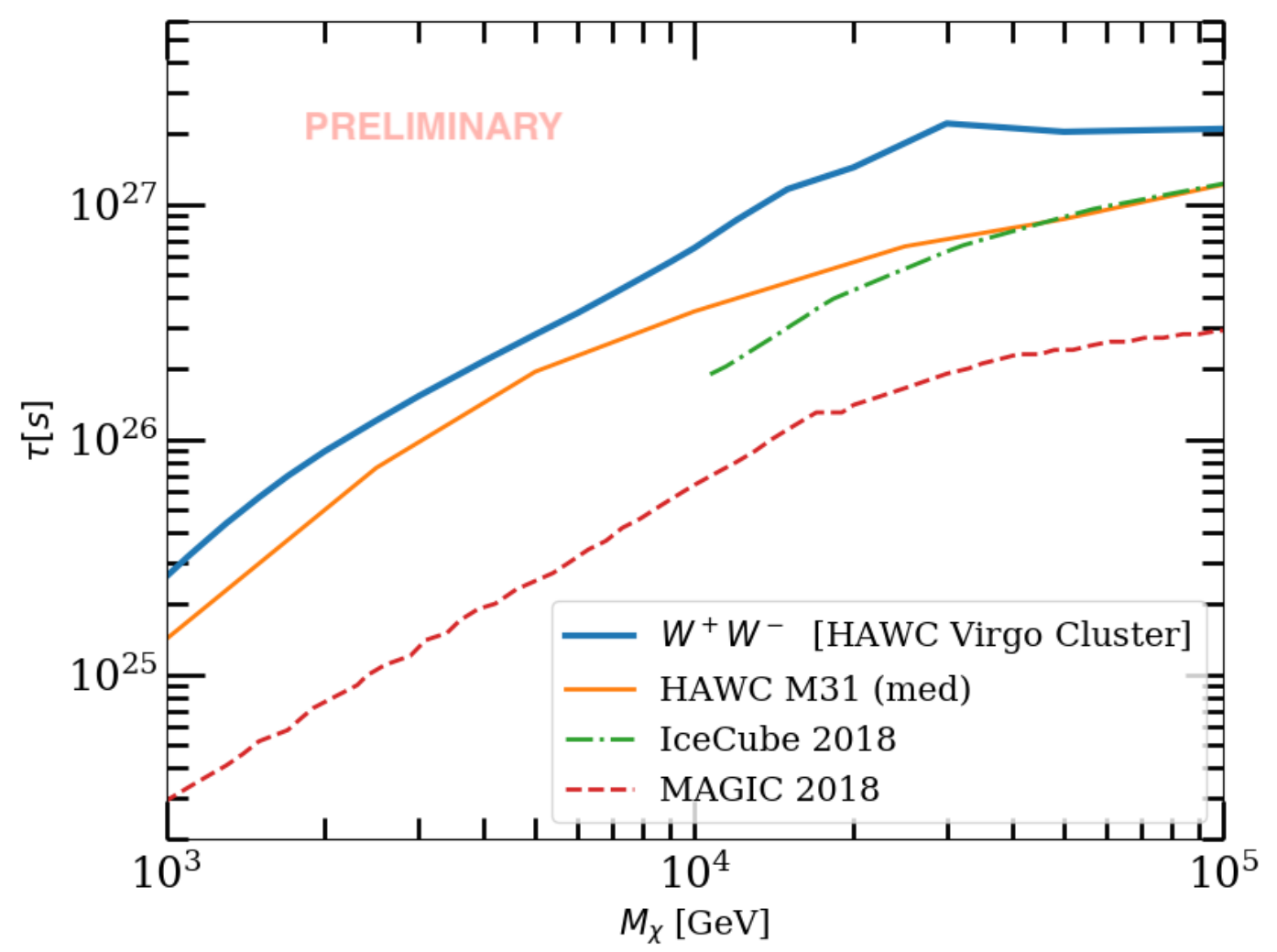

Figure 4: HAWC 95\% CL limits on the decay lifetime of dark matter decaying to $W^{+} W-$, assuming the spatial template parameters described in the text. Also shown are limits obtained using the M31 galaxy as a target with HAWC [8],MAGIC [10] and IceCube [11].

source hypothesis consists of the DM template with the expected flux calculated according to equation 1, in addition to a point source centered at the AGN M87. The point source at M87 is best-fit by a cut-off power-law spectrum, $\frac{d N}{d E} \sim\left(\frac{E}{E_{c}}\right)^{-i}$ with a spectral index $i$ of -1.8 and a cutoff energy $E_{c}$ of $6 \mathrm{TeV}$. The likelihood fits and limit calculation are performed using a generalized maximum likelihood Python package called Multi-Mission Maximum Likelihood (3ML) [19].

\section{Results}

Figures 2, 3 and 4 show the 95\% lower limits on the decay lifetime of DM. For comparison, we also show limits obtained in a similar work by HAWC using the M31 galaxy [8]. Also shown are the limits obtained by IceCube [11], Fermi-LAT [9] and the MAGIC collaboration using the Perseus cluster of galaxies [10]. As seen in the figures, HAWC limits are the strongest for the $W^{+} W^{-}$ channel across all masses. For $b \bar{b}$ and $\tau \bar{\tau}$, HAWC constraints are the strongest above $\sim 20 \mathrm{TeV}$. 


\section{Conclusions}

We search for gamma-ray emission from decaying DM correlated with the direction of galaxies in the Virgo cluster. Finding no significant excess, we set limits on the decay lifetime of the DM for three different channels. For $100 \mathrm{TeV}$, HAWC results constrain $\tau$ upto a few time $10^{27} \mathrm{~s}$ which are amongst the strongest constraints in the world for these masses.

\section{References}

[1] J. Conrad and O. Reimer Nat Phys 13 no. 3, (Mar., 2017) 224-231.

[2] B.-L. Young Frontiers of Physics 12 no. 2, (Oct., 2016) 121201.

[3] J. Liu, X. Chen, and X. Ji Nature Physics 13 (Mar., 2017) 212-216.

[4] HAWC Collaboration Collaboration, A. U. Abeysekara et al. Astrophys. J. 843 (July, 2017) 39.

[5] HAWC Collaboration, A. Albert et al. Astrophys. J. 905 no. 1, (2020) 76.

[6] S. Schindler, B. Binggeli, and H. Bohringer Astronomy and Astrophysics 343 (Mar., 1999) $420-438$.

[7] VERITAS Collaboration, V. A. Acciari et al. Astrophys. J. 716 (2010) 819-824.

[8] HAWC Collaboration, A. Albert et al. JCAP 06 (2018) 043. [Erratum: JCAP 04, E01 (2019)].

[9] M. Ackerman et al. The Astrophysical Journal 761 no. 2, (Nov, 2012) 91.

[10] MAGIC Collaboration, V. A. Acciari et al. Phys. Dark Univ. 22 (2018) 38-47.

[11] IceCube Collaboration, M. G. Aartsen et al. Eur. Phys. J. C 78 no. 10, (2018) 831.

[12] Cirelli et al. JCAP 03 (2011) 051. [Erratum: JCAP 10, E01 (2012)].

[13] M. Hütten, C. Combet, and D. Maurin Comput. Phys. Commun. 235 (2019) 336-345.

[14] J. Diemand, M. Kuhlen, and P. Madau Astrophys. J. 657 (2007) 262-270.

[15] J. F. Navarro, C. S. Frenk, and S. D. M. White Astrophys. J. 462 (1996) 563-575.

[16] Fermi-LAT Collaboration, M. Ackermann et al. Astrophys. J. 812 no. 2, (2015) 159.

[17] M. A. Sánchez-Conde and F. Prada Mon. Not. Roy. Astron. Soc. 442 no. 3, (2014) 2271-2277.

[18] A. Moliné, M. A. Sánchez-Conde, S. Palomares-Ruiz, and F. Prada Mon. Not. Roy. Astron. Soc. 466 no. 4, (2017) 4974-4990.

[19] G. Vianello, R. J. Lauer, P. Younk, L. Tibaldo, J. M. Burgess, H. Ayala, P. Harding, M. Hui, N. Omodei, and H. Zhou, "The Multi-Mission Maximum Likelihood framework (3ML)," 7, 2015. arXiv: 1507.08343 [astro-ph.HE]. 


\section{Full Author List: HAWC Collaboration}

A.U. Abeysekara ${ }^{48}$, A. Albert ${ }^{21}$, R. Alfaro ${ }^{14}$, C. Alvarez ${ }^{41}$, J.D. Álvarez ${ }^{40}$, J.R. Angeles Camacho $^{14}$, J.C. Arteaga-Velázquez ${ }^{40}$, K. P. Arunbabu ${ }^{17}$, D. Avila Rojas ${ }^{14}$, H.A. Ayala Solares ${ }^{28}$, R. Babu ${ }^{25}$, V. Baghmanyan ${ }^{15}$, A.S. Barber ${ }^{48}$, J. Becerra Gonzalez ${ }^{11}$, E. Belmont-Moreno ${ }^{14}$, S.Y. BenZvi $^{29}$, D. Berley ${ }^{39}$, C. Brisbois ${ }^{39}$, K.S. Caballero-Mora ${ }^{41}$, T. Capistrán ${ }^{12}$, A. Carramiñana ${ }^{18}$, S. Casanova ${ }^{15}$, O. Chaparro-Amaro ${ }^{3}$, U. Cotti ${ }^{40}$, J. Cotzomi ${ }^{8}$, S. Coutiño de León ${ }^{18}$, E. De la Fuente $^{46}$, C. de León ${ }^{40}$, L. Diaz-Cruz ${ }^{8}$, R. Diaz Hernandez ${ }^{18}$, J.C. Díaz-Vélez ${ }^{46}$, B.L. Dingus ${ }^{21}$, M. Durocher ${ }^{21}$, M.A. DuVernois ${ }^{45}$, R.W. Ellsworth ${ }^{39}$, K. Engel ${ }^{39}$, C. Espinoza ${ }^{14}$, K.L. Fan ${ }^{39}$, K. Fang ${ }^{45}$, M. Fernández Alonso ${ }^{28}$, B. Fick ${ }^{25}$, H. Fleischhack ${ }^{51,11,52}$, J.L. Flores ${ }^{46}$, N.I. Fraija ${ }^{12}$, D. Garcia ${ }^{14}$, J.A. García-González ${ }^{20}$, J. L. García-Luna ${ }^{46}$, G. García-Torales ${ }^{46}$, F. Garfias ${ }^{12}$, G. Giacinti $^{22}$, H. Goksu ${ }^{22}$, M.M. González ${ }^{12}$, J.A. Goodman ${ }^{39}$, J.P. Harding ${ }^{21}$, S. Hernandez ${ }^{14}$, I. Herzog ${ }^{25}$, J. Hinton ${ }^{22}$, B. Hona ${ }^{48}$, D. Huang ${ }^{25}$, F. Hueyotl-Zahuantitla ${ }^{41}$, C.M. Hui ${ }^{23}$, B. Humensky $^{39}$, P. Hüntemeyer ${ }^{25}$, A. Iriarte ${ }^{12}$, A. Jardin-Blicq ${ }^{22,49,50}$, H. Jhee ${ }^{43}$, V. Joshi ${ }^{7}$, D. $\mathrm{Kieda}^{48}$, G J. Kunde ${ }^{21}$, S. Kunwar ${ }^{22}$, A. Lara ${ }^{17}$, J. Lee ${ }^{43}$, W.H. Lee ${ }^{12}$, D. Lennarz ${ }^{9}$, H. León $\operatorname{Vargas}^{14}$, J. Linnemann ${ }^{24}$, A.L. Longinotti ${ }^{12}$, R. López-Coto ${ }^{19}$, G. Luis-Raya ${ }^{44}$, J. Lundeen ${ }^{24}$, K. Malone ${ }^{21}$, V. Marandon ${ }^{22}$, O. Martinez ${ }^{8}$, I. Martinez-Castellanos ${ }^{39}$, H. Martínez-Huerta ${ }^{38}$, J. Martínez-Castro $^{3}$, J.A.J. Matthews ${ }^{42}$, J. McEnery ${ }^{11}$, P. Miranda-Romagnoli ${ }^{34}$, J.A. Morales-Soto ${ }^{40}$, E. Moreno ${ }^{8}$, M. Mostafá ${ }^{28}$, A. Nayerhoda ${ }^{15}$, L. Nellen ${ }^{13}$, M. Newbold ${ }^{48}$, M.U. Nisa ${ }^{24}$, R. NoriegaPapaqui $^{34}$, L. Olivera-Nieto ${ }^{22}$, N. Omodei ${ }^{32}$, A. Peisker ${ }^{24}$, Y. Pérez Araujo ${ }^{12}$, E.G. Pérez-Pérez ${ }^{44}$, C.D. Rho $^{43}$, C. Rivière ${ }^{39}$, D. Rosa-Gonzalez ${ }^{18}$, E. Ruiz-Velasco ${ }^{22}$, J. Ryan ${ }^{26}$, H. Salazar ${ }^{8}$, F. Salesa Greus $^{15,53}$, A. Sandoval ${ }^{14}$, M. Schneider ${ }^{39}$, H. Schoorlemmer ${ }^{22}$, J. Serna-Franco ${ }^{14}$, G. Sinnis ${ }^{21}$, A.J. Smith ${ }^{39}$, R.W. Springer ${ }^{48}$, P. Surajbali ${ }^{22}$, I. Taboada ${ }^{9}$, M. Tanner ${ }^{28}$, K. Tollefson ${ }^{24}$, I. Torres ${ }^{18}$, R. Torres-Escobedo ${ }^{30}$, R. Turner ${ }^{25}$, F. Ureña-Mena ${ }^{18}$, L. Villaseñor ${ }^{8}$, X. Wang ${ }^{25}$, I.J. Watson ${ }^{43}$, T. Weisgarber ${ }^{45}$, F. Werner ${ }^{22}$, E. Willox ${ }^{39}$, J. Wood ${ }^{23}$, G.B. Yodh ${ }^{35}$, A. Zepeda ${ }^{4}$, H. Zhou ${ }^{30}$

${ }^{1}$ Barnard College, New York, NY, USA, ${ }^{2}$ Department of Chemistry and Physics, California University of Pennsylvania, California, PA, USA, ${ }^{3}$ Centro de Investigación en Computación, Instituto Politécnico Nacional, Ciudad de México, México, ${ }^{4}$ Physics Department, Centro de Investigación y de Estudios Avanzados del IPN, Ciudad de México, México, ${ }^{5}$ Colorado State University, Physics Dept., Fort Collins, CO, USA, ${ }^{6}$ DCI-UDG, Leon, Gto, México, ${ }^{7}$ Erlangen Centre for Astroparticle Physics, Friedrich Alexander Universität, Erlangen, BY, Germany, ${ }^{8}$ Facultad de Ciencias Físico Matemáticas, Benemérita Universidad Autónoma de Puebla, Puebla, México, ${ }^{9}$ School of Physics and Center for Relativistic Astrophysics, Georgia Institute of Technology, Atlanta, GA, USA, ${ }^{10}$ School of Physics Astronomy and Computational Sciences, George Mason University, Fairfax, VA, USA, ${ }^{11}$ NASA Goddard Space Flight Center, Greenbelt, MD, USA, ${ }^{12}$ Instituto de Astronomía, Universidad Nacional Autónoma de México, Ciudad de México, México, ${ }^{13}$ Instituto de Ciencias Nucleares, Universidad Nacional Autónoma de México, Ciudad de México, México, ${ }^{14}$ Instituto de Física, Universidad Nacional Autónoma de México, Ciudad de México, México, ${ }^{15}$ Institute of Nuclear Physics, Polish Academy of Sciences, Krakow, Poland, ${ }^{16}$ Instituto de Física de São Carlos, Universidade de São Paulo, São Carlos, SP, Brasil, ${ }^{17}$ Instituto de Geofísica, Universidad Nacional Autónoma de México, Ciudad de México, México, ${ }^{18}$ Instituto Nacional de Astrofísica, Óptica y Electrónica, Tonantzintla, Puebla, México, ${ }^{19}$ INFN Padova, Padova, Italy, ${ }^{20}$ Tecnologico de Monterrey, Escuela de Ingeniería y Ciencias, Ave. Eugenio Garza Sada 2501, Monterrey, N.L., 64849, 
México, ${ }^{21}$ Physics Division, Los Alamos National Laboratory, Los Alamos, NM, USA, ${ }^{22}$ MaxPlanck Institute for Nuclear Physics, Heidelberg, Germany, ${ }^{23}$ NASA Marshall Space Flight Center, Astrophysics Office, Huntsville, AL, USA, ${ }^{24}$ Department of Physics and Astronomy, Michigan State University, East Lansing, MI, USA, ${ }^{25}$ Department of Physics, Michigan Technological University, Houghton, MI, USA, ${ }^{26}$ Space Science Center, University of New Hampshire, Durham, NH, USA, ${ }^{27}$ The Ohio State University at Lima, Lima, OH, USA, ${ }^{28}$ Department of Physics, Pennsylvania State University, University Park, PA, USA, ${ }^{29}$ Department of Physics and Astronomy, University of Rochester, Rochester, NY, USA, ${ }^{30}$ Tsung-Dao Lee Institute and School of Physics and Astronomy, Shanghai Jiao Tong University, Shanghai, China, ${ }^{31}$ Sungkyunkwan University, Gyeonggi, Rep. of Korea, ${ }^{32}$ Stanford University, Stanford, CA, USA, ${ }^{33}$ Department of Physics and Astronomy, University of Alabama, Tuscaloosa, AL, USA, ${ }^{34}$ Universidad Autónoma del Estado de Hidalgo, Pachuca, Hgo., México, ${ }^{35}$ Department of Physics and Astronomy, University of California, Irvine, Irvine, CA, USA, ${ }^{36}$ Santa Cruz Institute for Particle Physics, University of California, Santa Cruz, Santa Cruz, CA, USA, ${ }^{37}$ Universidad de Costa Rica, San José , Costa Rica, ${ }^{38}$ Department of Physics and Mathematics, Universidad de Monterrey, San Pedro Garza García, N.L., México, ${ }^{39}$ Department of Physics, University of Maryland, College Park, MD, USA, ${ }^{40}$ Instituto de Física y Matemáticas, Universidad Michoacana de San Nicolás de Hidalgo, Morelia, Michoacán, México, ${ }^{41}$ FCFM-MCTP, Universidad Autónoma de Chiapas, Tuxtla Gutiérrez, Chiapas, México, ${ }^{42}$ Department of Physics and Astronomy, University of New Mexico, Albuquerque, NM, USA, ${ }^{43}$ University of Seoul, Seoul, Rep. of Korea, ${ }^{44}$ Universidad Politécnica de Pachuca, Pachuca, Hgo, México, ${ }^{45}$ Department of Physics, University of Wisconsin-Madison, Madison, WI, USA, ${ }^{46}$ CUCEI, CUCEA, Universidad de Guadalajara, Guadalajara, Jalisco, México, ${ }^{47}$ Universität Würzburg, Institute for Theoretical Physics and Astrophysics, Würzburg, Germany, ${ }^{48}$ Department of Physics and Astronomy, University of Utah, Salt Lake City, UT, USA, ${ }^{49}$ Department of Physics, Faculty of Science, Chulalongkorn University, Pathumwan, Bangkok 10330, Thailand, ${ }^{50}$ National Astronomical Research Institute of Thailand (Public Organization), Don Kaeo, MaeRim, Chiang Mai 50180, Thailand, ${ }^{51}$ Department of Physics, Catholic University of America, Washington, DC, USA, ${ }^{52}$ Center for Research and Exploration in Space Science and Technology, NASA/GSFC, Greenbelt, MD, USA, ${ }^{53}$ Instituto de Física Corpuscular, CSIC, Universitat de València, Paterna, Valencia, Spain

\section{Acknowledgements}

We acknowledge the support from: the US National Science Foundation (NSF); the US Department of Energy Office of High-Energy Physics; the Laboratory Directed Research and Development (LDRD) program of Los Alamos National Laboratory; Consejo Nacional de Ciencia y Tecnología (CONACyT), México, grants 271051, 232656, 260378, 179588, 254964, 258865, 243290, 132197, A1-S-46288, A1-S-22784, cátedras 873, 1563, 341, 323, Red HAWC, México; DGAPA-UNAM grants IG101320, IN111716-3, IN111419, IA102019, IN110621, IN110521; VIEP-BUAP; PIFI 2012, 2013, PROFOCIE 2014, 2015; the University of Wisconsin Alumni Research Foundation; the Institute of Geophysics, Planetary Physics, and Signatures at Los Alamos National Laboratory; Polish Science Centre grant, DEC-2017/27/B/ST9/02272; Coordinación de la Investigación Científica de la Universidad Michoacana; Royal Society - Newton Advanced Fellowship 180385; Generalitat Valenciana, grant CIDEGENT/2018/034; Chulalongkorn University's CUniverse (CUAASC) 
grant; Coordinación General Académica e Innovación (CGAI-UdeG), PRODEP-SEP UDG-CA499; Institute of Cosmic Ray Research (ICRR), University of Tokyo, H.F. acknowledges support by NASA under award number 80GSFC21M0002. We also acknowledge the significant contributions over many years of Stefan Westerhoff, Gaurang Yodh and Arnulfo Zepeda Dominguez, all deceased members of the HAWC collaboration. Thanks to Scott Delay, Luciano Díaz and Eduardo Murrieta for technical support. 